\title{
Danger of EBUS-TBNA in case of bronchogenic cyst: learning from a near-fatal intrapericardial and intrabronchial rupture
}

\author{
Giuseppe Mangiameli ${ }^{1 *}$, Imene Bouassida ${ }^{1}$, Nicolas Dürrleman ${ }^{2}$ and Françoise Le Pimpec-Barthes ${ }^{1,3}$ \\ ${ }^{1}$ Division of Thoracic Surgery and Lung Transplantation, Hôpital Européen Georges Pompidou, Assistance Publique-Hôpitaux de Paris, Paris, France \\ ${ }^{2}$ Hôpital privé les Franciscaines, 3 rue Jean Bouin, 30032 Nîmes Cedex 1, France \\ ${ }^{3}$ Centre de Recherche des Condeliers,INSERM UMR-S 1138, Sorbonne Université, USPC, Université de Paris, F-75006, France
}

\begin{abstract}
Despite the improvement in modern imaging and the large consensus in the direct surgical treatment of all bronchogenic cysts (BC), the introduction and spread of endobronchial ultrasonography-guided trans-bronchial needle aspiration (EBUS-TBNA) has changed several mainstays. Cases of aspiration of BC during the diagnostic workup or as a definitive therapeutic tool were found in literature for several years. We report the case of a 54-year-old patient presenting a subcarinal fluid mass who was submitted to EBUS-TBNA during the diagnostic workup causing a near-fatal intrapericardial and intrabronchial rupture.

In our experience given the severe complications related to the puncture of BC we contraindicate EBUS-TBNA in the diagnostic management of BCs because it is a rather risky exam. In all fit patients affected by $\mathrm{BC}$, surgery represents the best diagnostic and therapeutic tool.
\end{abstract}

\section{Introduction}

Cystic lesions of the mediastinum represent about $12 \%$ to $18 \%$ of all mediastinal lesions in adults. Bronchogenic cysts (BCs) are the commonest, constituting approximately $50 \%$ of all mediastinal cysts [1].

Although some cysts are asymptomatic and fortunately discovered, they can eventually produce compression or irritation on adjacent structures causing symptoms and potential serious complications (rupture, bleeding and malignant transformation). For these reasons the complete surgical excision is usually considered the treatment of choice [1].

The usual diagnostic management of mediastinal cyst includes chest radiography, computed tomography $(\mathrm{CT})$ and magnetic resonance imaging (MRI) of the chest.

Several authors have proposed Endobronchial ultrasound (EBUS) Transbronchial needle Aspiration (TBNA), used in lung cancer staging, for needle aspiration of BCs for diagnosis and treatment, performing a deep and complete aspiration to obtain a collapse of the lesion $[2,3]$.

This procedure remains controversial because of the risk of failure and complications. Below we report a case of $\mathrm{BC}$ with varying complications after EBUS-TBNA, including near-fatal rupture.

\section{Case Report}

A 54-year-old patient with a smoking history of 40 pack year presented a subcarinal fluid mass fortuitously discovered. Because of negative bronchoscopy, EBUS-TBNA was rapidly performed finding only non-tumour tissue. The subsequent PET scan did not show any hypermetabolism. A diagnosis of $\mathrm{BC}$ was evoked, and surgical indication was decided. Some days before surgery, the patient needed urgent surgical pericardial drainage for symptomatic pericardial pus-like effusion. Three days later, the patient presented a bronchial flooding leading to ARDS, which required urgent set-up of ECLS (Figure 1). Bronchoscopy showed a bronchial fistula with pus-like fluid inside both bronchial trees. After clinical stabilization, the BC was partially removed with fistula closure using myoplasty. Following 6 weeks in resuscitation unit and full recovery, the patient was referred to our center because of major recurrent $\mathrm{BC}$ which was removed (Figure 2). After three years the patient is in good clinical condition, no relapse has been detected.

\section{Discussion}

BCs represent the most common mediastinal cysts in adults, usually located in the middle mediastinum near the trachea or the main bronchi. Issuing from embryonic buds of future bronchial structures but disconnected from bronchial trees, the cyst wall is lined with bronchial mucosa. The mucous production is responsible for thick fluid content within the cyst with continuous lifelong growth. This pathophysiological knowledge justifies the gold standard treatment advocated by the first series in the 70's-80's which was the early and complete surgical excision regardless symptoms [1].

${ }^{\star}$ Correspondence to: Giuseppe Mangiameli, Division of Thoracic Surgery and Lung Transplantation, Georges Pompidou European Hospital, 20 rue Leblanc, 75908 Paris Cedex 15, France, Tel: +33 15609 2475; Fax: +33 1560933 80; E-mail: giuseppe.mangiameli@aphp.fr

Received: December 16, 2019; Accepted: December 27, 2019; Published: December 30, 2019 


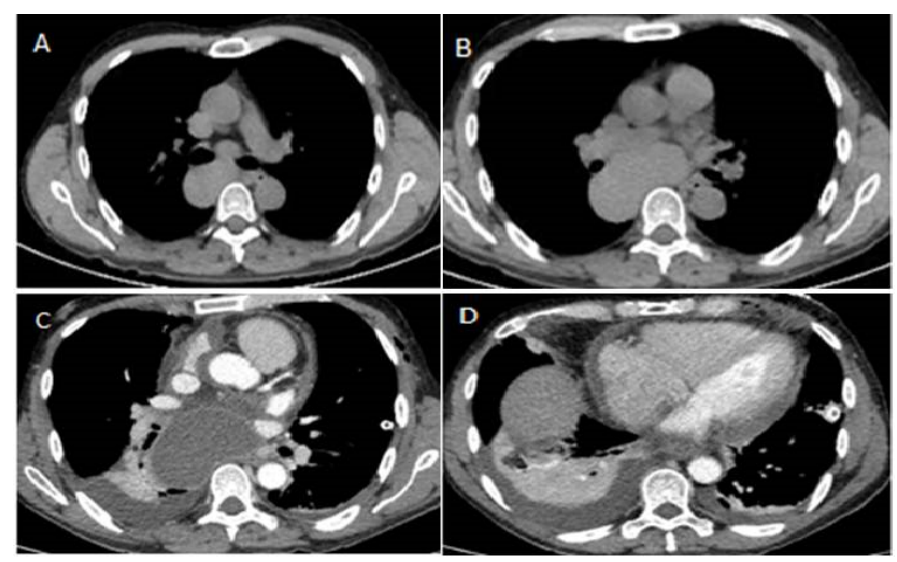

Figure 1. Chest computed tomography (CT) findings before and after EBUS guided needle aspiration (EBUS-TBNA). (A,B) a well-defined, homogeneous subcarinal cystic mass before EBUS-TBNA. (C,D) Contrast-enhanced CT 13 days after EBUS-TBNA showing infected pleural and pericardial effusion
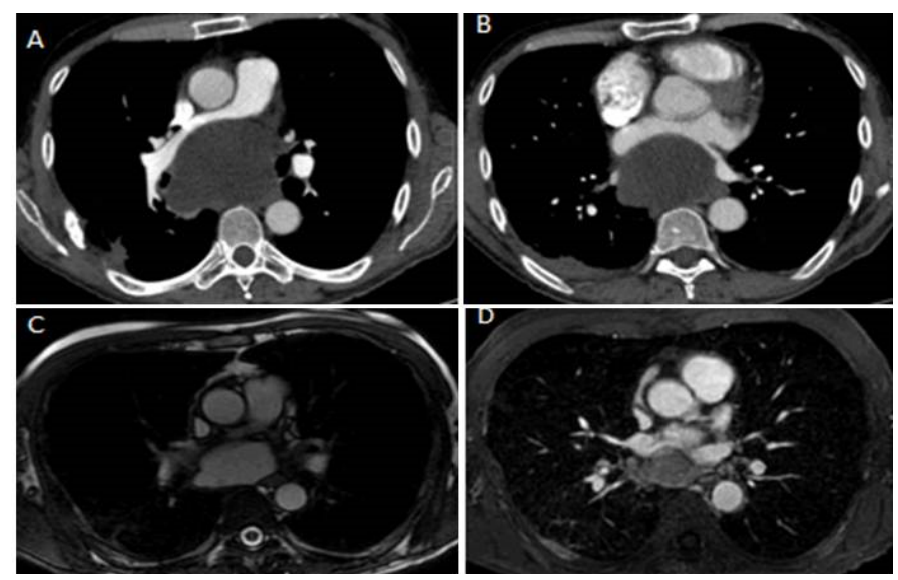

Figure 2. CT and MRI findings 4 months after EBUS-TBNA. CT and MRI images showing the cyst compressive effect on right pulmonary artery $(A, C)$ and left ventricle $(B, D)$

BCs are usually asymptomatic or potentially with non-specific respiratory symptoms. The compression on adjacent mediastinal structures is a risk of local complications and rare cases of malignant transformation have been reported.

The recent introduction of EBUS alongside the conventional radiological tools normally utilized in clinical practice (CT, RMI) has led to an extension of indications for BCs. In 1985 Schwartz, et al. proposed aspiration of BCs as a diagnostic modality, then surprisingly and without evaluation, EBUS -TBNA has been adopted in several diagnostic flow-charts [2]. More recently, Maturu, et al. has defined EBUS as a non-invasive modality of choice in diagnosing a $\mathrm{BC}$ and advocates EBUS-TBNA of BC as a potential therapeutic tool causing collapse of the cysts [3].

However, several complications have been repor[ted in literature since the increased worldwide spread of EBUS and the use of EBUSTBNA in determining the diagnosis of $\mathrm{BC}$ through aspiration.

The EBUS-dedicated bronchoscope passes through contaminated cavities (oral cavity and pharynx) and the aspiration causes a communication between airways and the fluid. Therefore, EBUSTBNA can easily be suspected of causing infection, but its role in mediastinitis is not clear. Existing mediastinal tissue between the bronchial and cyst walls could lead to mediastinitis after EBUS. Nowadays, nosocomial infection due to inappropriate bronchoscope disinfection or mishandling of devices has also been considered as possible causes of infectious complications.

Interestingly, several studies assert that when the puncture site is necrotic or cystic, there is a slight blood flow, decreased resistance and reduced bacterial attachment clearance. For these reasons, mediastinal cysts are more susceptible to infection compared to lymph nodes or nodules. In 2003, Wildi, et al. concluded that aspiration of suspected cysts should be undertaken with caution, given the high risk of infection [4]. More recently, a nationwide survey by the Japan Society for Respiratory Endoscopy has reported complications associated with EBUS-TBNA performed in Japan between January 2011 and June 2012. The authors reported a general complication rate of $1.23 \%$, and an infectious complication rate of $0.19 \%$ independent of EBUS-TBNA indication. They concluded that the puncture of necrotic or cystic areas should be avoided [5].

Based on the aforementioned case and the reported literature, EBUS-TBNA may not be as safe as previously thought. In particular, several doubts remain about the systematic aspiration of BCs during the diagnostic workup especially when conventional radiology allows high certitude of $\mathrm{BC}$ diagnosis.

Large surgical series confirmed that in symptomatic patients submitted to surgical resection of BCs short-term results are excellent, operative morbidity is acceptable and symptoms disappear [1]. Similarly, a surgical approach should be considered even in asymptomatic patients for three reasons. First of all, because several series showed that BCs often become symptomatic with long-term follow-up and complications can develop when observed. Secondly, symptom onset makes surgery more difficult until to be a challenge in the case of infection. Lastly, while surgical removal of a simple BC is relatively easy with minimal morbidity, the advent of VATS shortens the time of disability and hospitalization.

The use of EBUS-TBNA should probably be considered in several specific cases as an alternative to surgery in adults with BCs unfit or reluctant to undergo surgery. However, the strongest indication for surgery remains prevention of malignant transformation of $\mathrm{BCs}$, a rare but possible occurrence. The choice of a therapeutic EBUS-TBNA should consider this occurrence and the high rate of recurrence. Cyst aspiration does not allow mucosal lining removal which represents the milestone to avoid relapse. Finally, only surgery allows for a definitive histological diagnosis. In our opinion surgery remains the best diagnostic and therapeutic tool in all fit patients affected by BCs.

\section{References}

1. Takeda S, Miyoshi S, Minami M, Ohta M, Masaoka A, et al. (2003) Clinical spectrum of mediastinal cysts. Chest 124:125-132. [Crossref]

2. Schwartz DB, Beals TF, Wimbish KJ, Hammersley JR (1985) Transbronchial fine needle aspiration of bronchogenic cysts. Chest 88: 573-575. [Crossref]

3. Maturu VN, Dhooria S, Agarwal R (2015) Efficacy and Safety of Transbronchial Needle Aspiration in Diagnosis and Treatment of Mediastinal Bronchogenic Cysts. $J$ Bronchol Intervent Pulmonol 22: 195-203. [Crossref]

4. Wildi SM, Hoda RS, Fickling W, Schmulewitz N, Varadarajulu S, et al. (2003) Diagnosis of benign cysts of the mediastinum: the role and risks of EUS and FNA. Gastrointest Endosc 58: 362-368. [Crossref]

5. Asano F, Aoe M, Ohsaki Y, Okada Y, Sasada S, et al. (2013) Complications associated with endobronchial ultrasound-guided transbronchial needle aspiration: a nationwide survey by the Japan Society for Respiratory Endoscopy. Respiratory Research 14: 50. [Crossref]

Copyright: (C2019 Mangiameli G. This is an open-access article distributed under the terms of the Creative Commons Attribution License, which permits unrestricted use, distribution, and reproduction in any medium, provided the original author and source are credited. 